\title{
Comparative analysis of mesenchymal stem cell and embryonic tendon progenitor cell response to embryonic tendon biochemical and mechanical factors
}

Jeffrey P Brown ${ }^{1}$, Thomas V Galassi ${ }^{1}$, Matteo Stoppato ${ }^{1}$, Nathan R Schiele ${ }^{1}$ and Catherine K Kuo ${ }^{1,2^{*}}$

\begin{abstract}
Introduction: Advances in tendon engineering with mesenchymal stem cells (MSCs) are hindered by a need for cues to direct tenogenesis, and markers to assess tenogenic state. We examined the effects of factors involved in embryonic tendon development on adult MSCs, and compared MSC responses to that of embryonic tendon progenitor cells (TPCs), a model system of tenogenically differentiating cells.

Methods: Murine MSCs and TPCs subjected to cyclic tensile loading, transforming growth factor- $\beta 2$ (TGF $\beta 2$ ), and fibroblast growth factor-4 (FGF4) in vitro were assessed for proliferation and mRNA levels of scleraxis, TGF $\beta 2$, tenomodulin, collagen type I and elastin.

Results: Before treatment, scleraxis and elastin levels in MSCs were lower than in TPCs, while other tendon markers expressed at similar levels in MSCs as TPCS. TGF $\beta 2$ alone and combined with loading were tenogenic based on increased scleraxis levels in both MSCs and TPCs. Loading alone had minimal effect. FGF4 downregulated tendon marker levels in MSCs but not in TPCs. Select tendon markers were not consistently upregulated with scleraxis, demonstrating the importance of characterizing a profile of markers.

Conclusions: Similar responses as TPCs to specific treatments suggest MSCs have tenogenic potential. Potentially shared mechanisms of cell function between MSCs and TPCs should be investigated in longer term studies.
\end{abstract}

\section{Introduction}

Tendons transmit muscle-derived forces to bone to enable skeletal movement. Unfortunately, these tissues suffer $\sim 15$ million musculoskeletal injuries annually in the USA [1]. Due to the poor innate healing ability of tendons, surgical intervention is the primary approach to repairing injured tendon despite substantial failure rates, limited long-term function recovery, donor site morbidity with autologous transplants, and risk of infections $[2,3]$. These significant drawbacks have motivated efforts to engineer replacement tendon with mesenchymal stem cells (MSCs) [4-9].

\footnotetext{
* Correspondence: CatherineK.Kuo@tufts.edu

'Department of Biomedical Engineering Tufts University, Science and

Technology Center, 4 Colby Street, Medford, MA 02155, USA

${ }^{2}$ Cell, Molecular \& Developmental Biology Program Sackler School of Graduate Biomedical Sciences, Tufts University School of Medicine, 145 Harrison Avenue, Boston, MA 02111, USA
}

Adult MSCs are attractive for tissue regeneration strategies as they have the potential to differentiate toward various musculoskeletal lineages, including osteogenic, chondrogenic and adipogenic, in response to established lineage-specific cues. However, such cues have not been identified for tenogenic differentiation, and tissue engineering approaches to tenogenically differentiate MSCs have not achieved functional tendons [4-14]. This may be in part because evaluation of tenogenic differentiation is challenged by limited knowledge of how tenogenically differentiating cells should behave. Scleraxis (Scx) is the only known tendon-specific marker that is expressed during early development and sustained throughout tissue formation [15]. However, Scx expression levels do not vary in embryonic tendon progenitor cells (TPCs) between developmental stages [16]. Furthermore, mice with a mutation in the Scx gene have defects in only a subset of tendons, indicating $S c x$ is not a master 


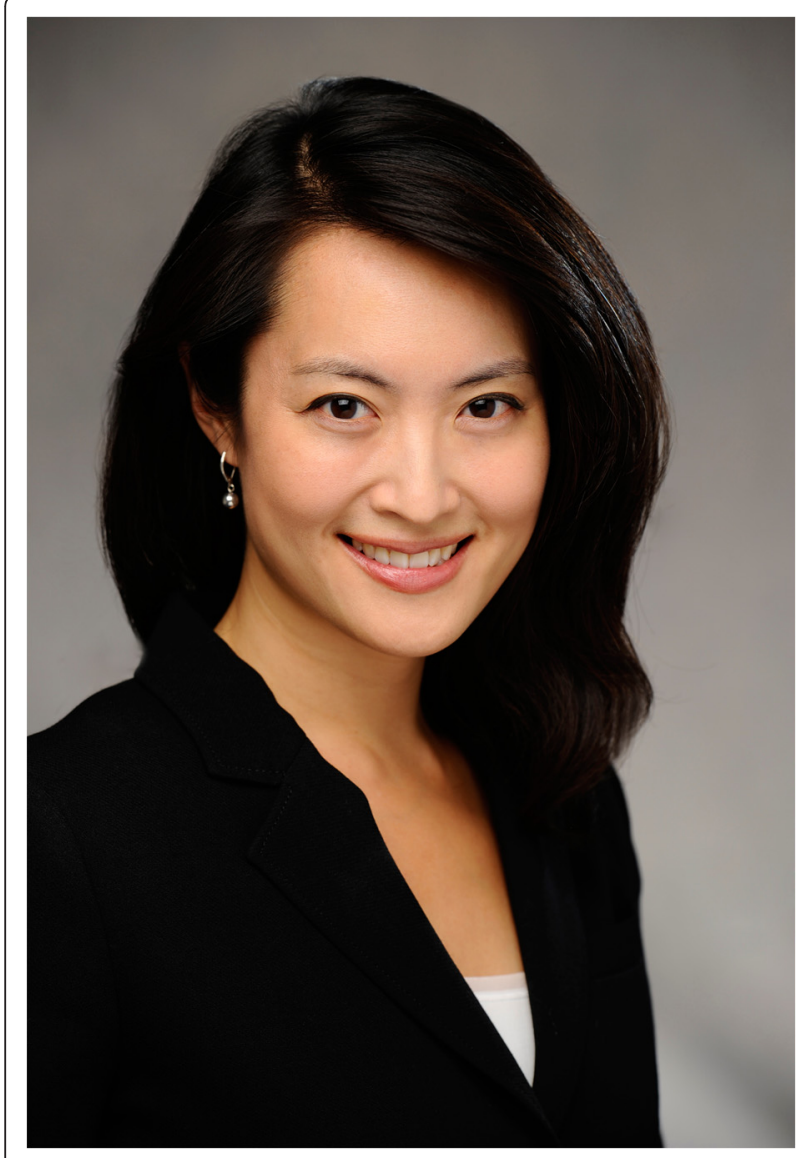

Box 1. About Catherine K. Kuo

CKK is an Assistant Professor of Biomedical Engineering at Tufts University, and a faculty member of the Cell, Molecular and Developmental Biology Program in the Sackler School of Graduate Biomedical Sciences at the Tufts University School of Medicine. She earned a BSE in Materials Science and Engineering and a PhD in Biomaterials and Macromolecular Science and Engineering from the University of Michigan, and pursued postdoctoral training in the Cartilage Biology and Orthopaedics Branch of the NIAMS at the National Institutes of Health. Her research focuses on developing adult stem cell differentiation strategies informed by embryogenesis. Specifically, she is identifying mechanical and chemical properties of embryonic tissue microenvironments that can be presented via biomaterials and bioreactor cultures to direct stem cell behavior. With this approach, she is designing replacement tissues for regenerative medicine, and also engineering tissue models as platforms to investigate mechanisms of embryonic tissue formation and wound healing.

regulator of tendon differentiation [17]. Recognizing these limitations, we recently examined how a profile of tendon markers, including Scx, late marker tenomodulin (Tnmd), and other relevant but non-specific markers (transforming growth factor (TGF) 32 , collagen type I (Col I) and elastin (Eln)), respond to embryonic tendon cues [16].

We identified TGF 32 , and combinations with fibroblast growth factor-(FGF)4 and loading, as potential in vitro tenogenic cues based on upregulation of Scx and modulation of other tendon markers in embryonic TPCs, a model system of tenogenically differentiating cells [16]. Understanding how embryonic progenitor cells respond to developmental factors has been successful in establishing stem cell differentiation programs for other lineages. For example, protocols to direct chondrogenesis of adult MSCs are based on methods that utilize embryonic cartilage development factors to chondrogenically differentiate embryonic mesenchymal limb bud cells $[18,19]$. Factors to guide stem cell differentiation are selected based on their ability to induce marker expression patterns similar to that exhibited temporally by embryonic mesenchymal progenitor cells during development [20-25]. In contrast, how MSCs respond to treatments in comparison with embryonic cells that are committed to the tendon lineage (that is, TPCs) has not been investigated.

The need for mechanical loading for adult tendon homeostasis has motivated application of dynamic tensile loading as a primary cue to tenogenically differentiate MSCs. However, reports on the effectiveness of loading on tenogenesis have been inconsistent $[6-8,10,26]$, and thus the efficacy of dynamic tensile loading to tenogenically differentiate MSCs is unclear. Developmentally, mechanical loading seems critical for tendon formation $[27,28]$, as muscle paralysis during embryonic chick development resulted in malformed tendons [29-31]. However, paralysis may also have contributed to aberrant tendon formation by altering soluble factors secreted by muscle, such as FGF4 [32,33]. We reported mechanical loading alone had little effect on embryonic TPC behavior, but that specific loading and growth factor combinations differentially regulated tendon marker gene expression [16]. Interactions between growth factors and dynamic loading could play a key role in tenogenesis.

Tendon engineering strategies with MSCs have used growth factors involved in adult tendon wound healing $[13,14]$, including TGF $\beta 1$, insulin-like growth factor, platelet-derived growth factor, epidermal growth factor, and FGF2 [34], despite their potential roles in the formation of scarred tendon with aberrant biochemical composition, organization and mechanical properties [35]. In contrast, embryonic tendon development involves different factors, including FGF4 and TGF $\beta 2$ [32,33,36-38]. Though we demonstrated FGF4 and TGF $\beta 2$ influence embryonic TPC activity [16], the ability for these factors to tenogenically differentiate adult MSCs has not been reported. 
We hypothesized that MSCs would mimic TPCs in their response to tendon development factors. To test this hypothesis, we treated mouse adult MSCs and embryonic day (E) 14 TPCs with combinations of TGF $\beta 2$, FGF4 and mechanical loading, and assessed proliferation and gene expression. Our findings provide insight into MSC tenogenic potential and the utility of embryonic tendon factors to guide adult MSC differentiation toward a tenogenic lineage in vitro.

\section{Methods}

All materials were from Invitrogen (Carlsbad, CA, USA) unless otherwise specified.

Adult mouse bone marrow mesenchymal stem cell harvest Four-month-old male Scx-green fluorescent protein (GFP) mice [39] were sacrificed by $\mathrm{CO}_{2}$ asphyxiation and decapitation with Tufts University Institutional Animal Care and Use Committee approval. The hind limbs were skinned, and femurs and tibias were dissected and washed in sterile phosphate-buffered saline (PBS) without $\mathrm{MgCl}_{2} /$ $\mathrm{CaCl}_{2}$. Bone ends were removed and marrow was flushed with PBS. Cell suspensions were treated with red blood cell lysis buffer (Roche, Indianapolis, IN, USA), pelleted, washed with PBS, and resuspended in growth medium (GM) of Dulbecco's modified Eagle medium with 10\% fetal bovine serum (FBS) and $1 \%$ penicillin/streptomycin. Cells were plated at $1 \times 10^{6}$ cells $/ \mathrm{cm}^{2}$ and cultured at $37^{\circ} \mathrm{C}$ and $5 \% \mathrm{CO}_{2}$. Three independent MSC pools, isolated by plastic adherence [7], were expanded to passage 3.

\section{Embryonic mouse tendon progenitor cell harvest}

E14 embryos were harvested from pregnant Scx-GFP mice and staged [40] with Tufts University Institutional Animal Care and Use Committee approval. Limbs were isolated, minced, incubated under agitation at $200 \mathrm{rpm}$ in $1 \%$ type II collagenase in PBS at $37^{\circ} \mathrm{C}$ for 45 minutes, and neutralized with GM. Cell suspensions were passed through a 40- $\mu \mathrm{m}$ cell strainer (BD Biosciences, San Jose, CA, USA), pelleted, washed in PBS, re-suspended in GM, plated at $1 \times 10^{4}$ cells $/ \mathrm{cm}^{2}$, and cultured at $37^{\circ} \mathrm{C}$ and $5 \% \mathrm{CO}_{2}$. Three independent limb cell pools were harvested. Cells were trypsinized when $80 \%$ confluent and sorted on the basis of GFP signal using a MoFlo Legacy cell sorter (Beckman Coulter, Brea, CA, USA) at $488 \mathrm{~nm}$ excitation and collected by a 530/40 filter. TPCs were expanded to passage $1-2$.

\section{Growth factor treatment and mechanical loading}

TPCs and MSCs were seeded at $2 \times 10^{4}$ cells $/ \mathrm{cm}^{2}$ on Col I-coated Uniflex ${ }^{\odot}$ plates (Flexcell International, Hillsborough, NC, USA) and incubated in GM for cell attachment. After 48 hours (day (D)0), GM was replaced with basal (control) medium (BM; Dulbecco's modified Eagle medium, 1\% FBS and 1\% penicillin/streptomycin), or BM supplemented with $100 \mathrm{ng} / \mathrm{mL}$ rhFGF4 and/or 1 ng/mL rhTGF $\beta 2$ (PeproTech, Rocky Hill, NJ, USA), and cyclically loaded under uniaxial tension with $1 \%$ sinusoidal strain at $0.5 \mathrm{~Hz}$ for 1 hour/day, as previously described [16]. Static controls were treated identically, without cyclic loading. Medium was replaced after 48 hours.

\section{Cell proliferation}

Cells were fixed for 20 minutes in $10 \%$ phosphatebuffered formalin, stained with 4',6-diamidino-2-phenylindole nucleic acid dye, and imaged using an inverted Leica DM IL fluorescent microscope and DFC340 FX camera (Leica Microsystem, Buffalo Grove, IL, USA). Three fields per well (left, middle, and right) were imaged for each condition and cell pool. Nuclei were counted using Image J (National Institutes of Health, Bethesda, MD, USA).

\section{Quantitative polymerase chain reaction}

MSCs and TPCs were harvested on D0 and D3 for RNA isolation. Cells were homogenized in TRIzol reagent and total RNA was isolated. Samples were reversetranscribed using the Superscript III First Strand Synthesis kit. Quantitative (q)PCR was performed with Brilliant II SYBR Green qPCR master mix (Agilent, Wilmington, DE, USA) on a Stratagene Mx3000P multiplex qPCR system (Agilent). Previously optimized mouse-specific primers for Scx, Tnmd, Col I, Eln, TGF $\beta 2$ and $18 \mathrm{~s}$ were used [16]. Fold change was calculated as $2^{-\Delta \Delta C T}$.

\section{Statistical analysis}

Results were obtained from three independent cell pools and are shown as mean \pm standard deviation. Statistical analyses were performed using GraphPad Prism (GraphPad Software Inc., San Diego, CA, USA). Treatment effects were evaluated using a two-way analysis of variance with Tukey's post-hoc test or Student's $t$-test, and considered statistically significant when $P<0.05$. qPCR data were log-transformed before statistical analysis and plotted as fold difference values $\left(2^{-\Delta \Delta C T}\right)$.

\section{Results}

\section{Effects of treatments on mesenchymal stem cells}

MSC number did not change with treatment or time $(P>0.05$; Figure 1A). MSCs appeared fibroblastic with all treatments (not shown). On D3, Scx was downregulated by FGF4 and FGF4 + loading, but upregulated by TGF $\beta 2$ and TGF $\beta 2$ + loading $(P<0.05$; Figure $2 \mathrm{~A})$. FGF4 combinations downregulated TGF $32 \quad(P<0.05$; Figure 2B). All combinations, except loading alone, downregulated Tnmd $(P<0.05$; Figure $2 C)$. FGF4 and FGF4 + loading downregulated Col I $(P<0.05)$ TGF $\beta 2$ 

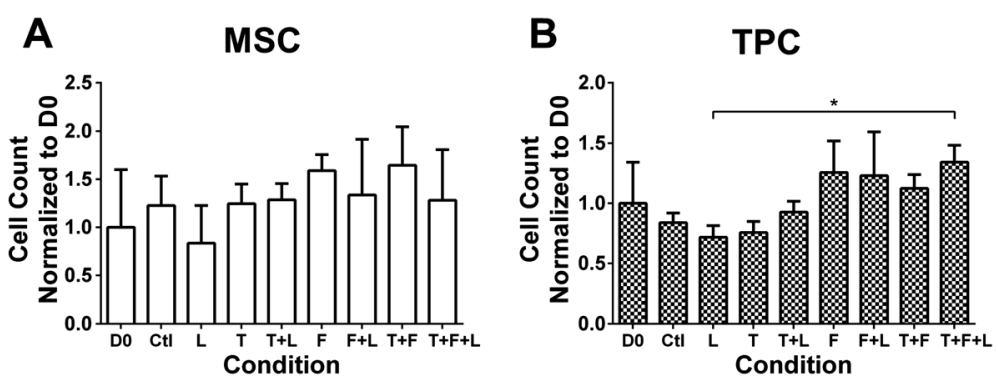

Figure 1 Mesenchymal stem cell (MSC) and tendon progenitor cell (TPC) proliferation as a function of growth factor treatments and loading. Effects on MSC and TPC proliferation on day (D)3 (normalized to D0) of treatment with combinations of mechanical loading (L), transforming growth factor (TGF) 32 (T), and fibroblast growth factor-(FGF)4 (F) treatment. Left column shows D0 data. (A) MSC proliferation was not significantly affected by any treatment. (B) TPC proliferation was not significantly affected by any treatment, but there was a significant difference between loading and TGF $32+$ FGF4 + loading groups on D3. ${ }^{*} P<0.05$.

combinations caused Col I to trend up $(P>0.05$; Figure 2D), with TGF $\beta 2$ + loading approaching significance $(P=0.06)$. All treatments downregulated Eln at D3 compared to control $(P<0.05$; Figure 2E).

\section{Effects of treatments on tendon progenitor cells}

TPC number did not change with treatment or time $(P>0.05$; Figure 1B), though was higher for TGF $32+$ FGF4 + loading compared to loading alone on D3 $(P<0.05$; Figure 1B). TPCs appeared fibroblastic with all treatments (not shown). On D3, Scx was upregulated by TGF $\beta 2$ combinations $(P<0.05)$, but was not affected by loading, FGF4, or FGF4 + loading (Figure 3A). TGF 32 and Tnmd were downregulated by TGF $\beta 2+$ FGF 4 + loading $(P<0.05$; Figure $3 \mathrm{~B}, \mathrm{C})$, and exhibited similar expression patterns with all treatments (Figure 3B,C). Col I was upregulated by TGF 32 + loading $(P<0.01)$, but not affected by other treatments (Figure 3D). Eln was downregulated by FGF4 combinations, but upregulated by TGF $\beta 2+$ loading $(P<0.05$; Figure 3E).

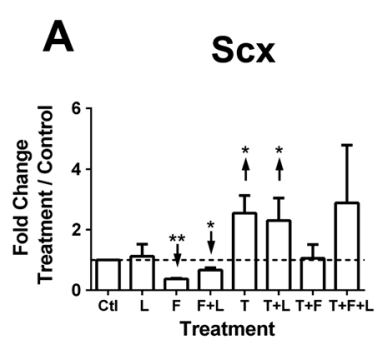

B TGF 22
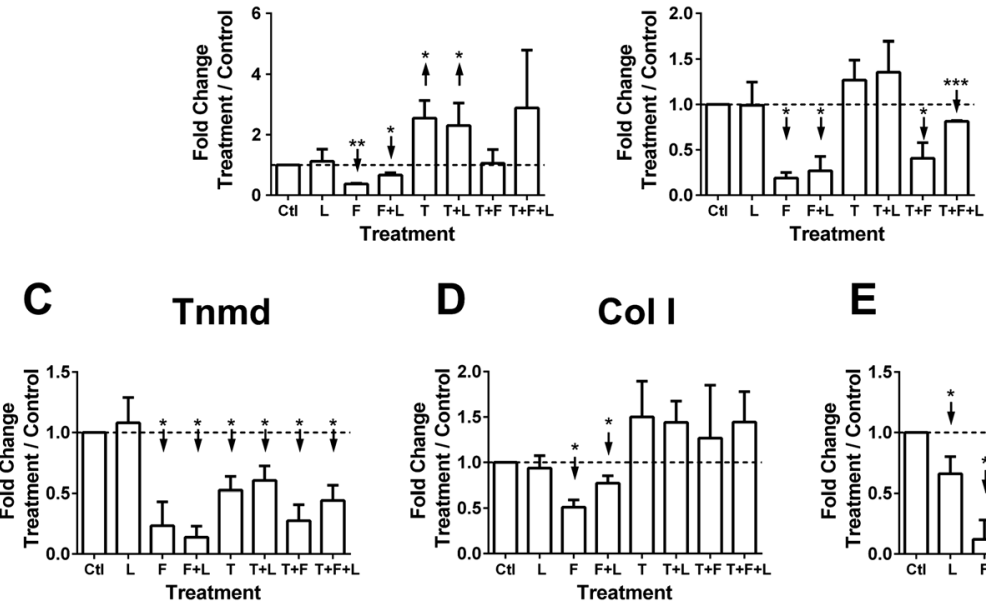

D Col I

E

Eln expression on day (D)3 of treatment with combinations of mechanical loading (L), transforming growth factor (TGF) $\beta 2$ (T), and fibroblast growth factor-(FGF)4 (F). Dashed horizontal line $=1$ indicates control condition. (A) Scleraxis (SCX) was significantly downregulated by FGF4 and FGF4 + loading, and upregulated by TGF 32 and TGF $\beta 2$ + loading. (B) TGF 32 was significantly downregulated by all treatments involving FGF4. (C) All treatments except loading significantly downregulated tenomodulin (Tnmd). (D) Collagen type I (Col I) was significantly downregulated by FGF4 and FGF4 + loading, while all treatments involving TGF 32 caused Col I to trend up $(P \geq 0.06)$. (E) Elastin (EIn) was significantly downregulated by all treatments. $\uparrow$ or $\downarrow$ indicates statistically significant up- or downregulation, respectively; ${ }^{*} P<0.05,{ }^{* *} P<0.01$, ${ }^{* * *} P<0.001$. 


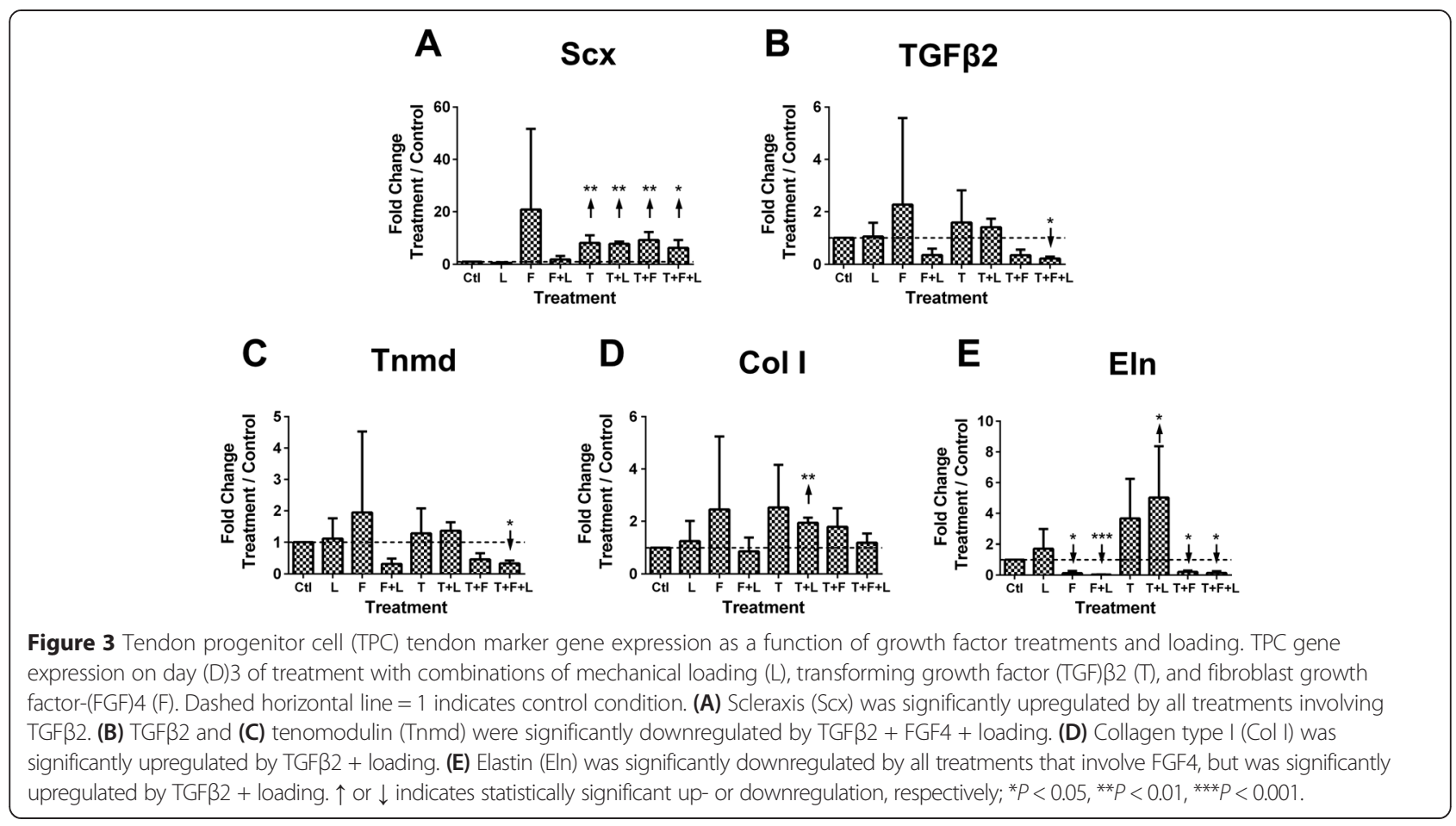

\section{Comparison of mesenchymal stem cell and tendon progenitor cell gene expression}

Under control conditions and when loaded, Eln expression in MSCs increased $>20$-fold from D0 to D3 $(P<0.001$; Figure 4A). Other treatment combinations had inhibitory effects on this upregulation of Eln expression (Figure 4A). In TPCs, Eln expression increased $>15$-fold from D0 to D3 in control culture and with loading $(P<0.05$; Figure 4B). This increase was abrogated by FGF4 combinations. In contrast to MSCs, TGF $\beta 2$ and TGF $\beta 2+$ loading enhanced Eln expression in TPCs from D0 to D3 $(P<0.01$; Figure 4B). Expression of other genes did not vary with time (not shown).
To investigate baseline differences between MSCs and TPCs, tendon marker expression levels were compared at D0. Scx and Eln expression in MSCs were lower (61fold and 138-fold, respectively) than in TPCs $(P<0.05$; Figure 5A). However, TGF $\beta 2$, Tnmd, and Col I levels were similar between TPCs and MSCs $(P=0.82, P=0.46$ and $P=0.36$, respectively; Figure $5 \mathrm{~A})$. To assess tenogenic potential of MSCs, MSC response to TGF $\beta 2$ was compared to that of TPCs at D3 (Figure 5B-F). TGF 2 was chosen as a tenogenic factor for upregulating Scx in TPCs. On D3 of TGF $\beta 2$ treatment, Scx, Col I, TGF $\beta 2$, Tnmd and Eln expression trended up in both MSCs and TPCs, compared to D0. MSCs and TPCs were not

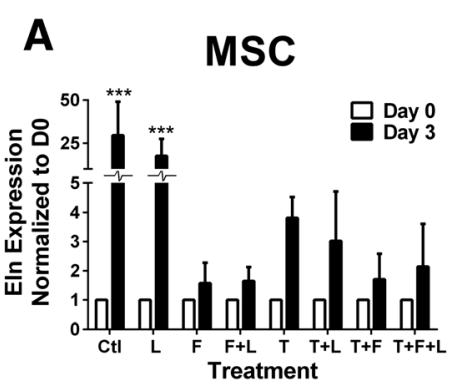

B TPC

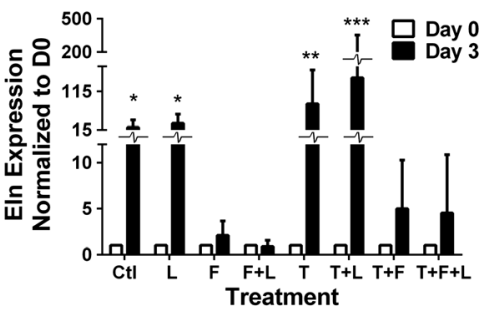

Figure 4 Elastin (EIn) gene expression as a function of growth factor treatments and loading. Eln gene expression in mesenchymal stem cells (MSCs) and tendon progenitor cells (TPCS) on day (D)3 of treatment with combinations of mechanical loading (L), transforming growth factor (TGF) $\beta 2$ (T), and fibroblast growth factor-4 (F), and normalized to D0. (A) MSCs significantly increased Eln with time in control culture and with loading. (B) TPCs significantly increased Eln with time in control culture and treatment with loading, TGF $\beta 2$, and TGF $\beta 2+$ loading. $\uparrow$ or $\downarrow$ indicates statistically significant up- or downregulation, respectively; ${ }^{*} P<0.05,{ }^{* *} P<0.01,{ }^{* * *} P<0.001$. 

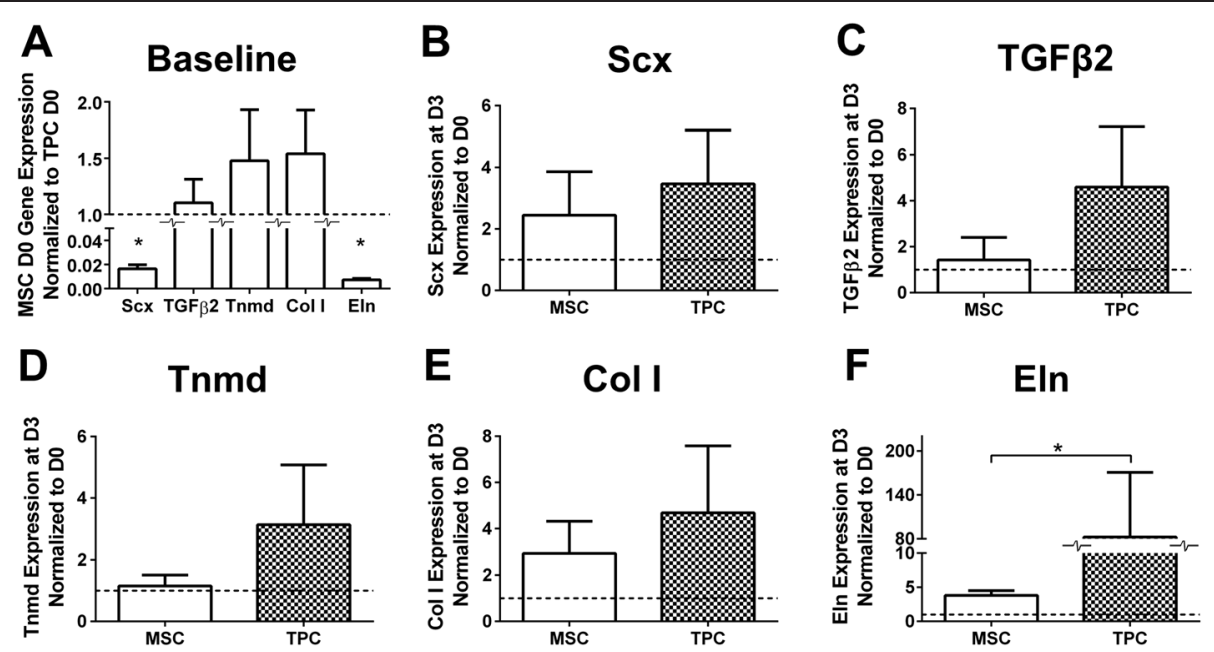

Figure 5 Comparison of mesenchymal stem cell (MSC) and tendon progenitor cell (TPC) tendon marker gene expression at baseline and with transforming growth factor (TGF) 32 treatment. Comparison of MSC and TPC gene expression at day (D)0 and D3 of TGF $\beta 2$ treatment. (A) Comparison of tenogenic gene expression by MSCS versus TPCs at D0; scleraxis (SCX) and elastin (Eln) were significantly lower in MSCs compared to TPCS. At D3 (normalized to D0) of TGF 32 treatment, (B) SCX, (C) TGF 32 , (D) tenomodulin (Tnmd), and (E) collagen type I (Col I) were not significantly different between MSCs and TPCs, while (F) Eln was significantly higher in TPCs than MSCs. ${ }^{P} P<0.05$.

significantly different in Scx $(P=0.54)$, Col I $(P=0.39)$, TGF $\beta 2(P=0.13)$ and Tnmd $(P=0.17)$ levels (Figure 5B-E), but the TGF 32 -induced increases in Eln were 21-fold greater in TPCs than in MSCs $(P<0.05$; Figure 5F).

\section{Discussion}

To date, a standard protocol to tenogenically differentiate MSCs has not been established. A major challenge is a need for potent tenogenic factors. Another significant obstacle is a lack of metrics to assess tenogenic state. We characterized the effects of embryonic tendon cues on adult MSCs in comparison with embryonic TPCs. Utilizing murine cells enabled investigation of embryonic TPCs, which would not be possible with human cells. Additionally, the murine system develops more rapidly than human. Previous work demonstrated chondrogenic growth factors induced murine embryonic limb bud cells to form Sox9-expressing aggregates in 24 hours, and sulfated proteoglycan-rich cartilaginous nodules by 3 days [41]. Here, embryonic tendon development factors influenced both MSCs and E14 TPCs within 3 days. At E14, TPCs have just condensed into overt tendon tissue forms in vivo [42], and embryonic muscle-induced movements that could impose mechanical stimulation on developing tendon are first observed [43]. Our results suggest MSCs have tenogenic potential, based on similar responses as E14 TPCs when subjected to tenogenic treatments.

MSCs and E14 TPCs were examined at D0 to compare baseline gene expression profiles (Figure 5). At D0, cells have been expanded in vitro but not subjected to treatments, representing a starting point in many tissue engineering strategies. Tnmd and Col I are late markers of tendon development, so it was expected that MSCs and TPCs in early differentiation stages expressed both genes similarly. Lower baseline Scx levels in MSCs compared to E14 TPCs suggest MSCs are inherently less committed to the tenogenic lineage than TPCs, while similar TGF 32 and Tnmd levels may reflect that E14 TPCs and MSCs are both at an immature stage of differentiation. TGF $\beta 2$ may be a tendon marker as well as a tenogenic cue. TGF $\beta 2$ is present in embryonic chick tendons [36], expressed in murine E12.5 to E15.5 limb tendons $[37,44]$, and can upregulate Scx expression in embryonic murine limbs in vivo [37]. Additionally, TGF $\beta 2^{-/-}$mice possess tendon defects [37].

Cyclic loading alone did not upregulate most tendon markers examined, but increased Scx and Col I expression when combined with TGF $\beta 2$ (Figures 2 and 3). Our study was performed with $1 \%$ FBS. In contrast, studies in which dynamic loading enhanced Scx and Col I expression in MSCs used significantly higher serum levels $[6,8,10,26]$. Loading likely interacted with soluble factors from the serum to influence cell behavior in those studies. It is also possible that different loading parameters could yield different results, though others found that changing duty cycle did not affect MSC gene expression of Col I and other extracellular matrix components [4]. Furthermore, we characterized gene expression, which may not reflect changes at the protein level. We previously found cyclic loading enhanced collagen production by human MSCs in three-dimensional scaffolds without altering collagen mRNA levels [7]. Future studies that 
incorporate three-dimensional culture systems and assess protein level changes will be important.

Both cell types did not change in cell number with treatments (Figure 1) suggesting the treatments were possibly influencing cell functions other than proliferation, such as differentiation. TGF $\beta 2$ and TGF $\beta 2$ + loading induced higher Scx expression in both MSCs and TPCs when compared to control conditions on D3, though to different levels (Figures 2A and 3A). Conditions that upregulated Scx did not consistently upregulate other tendon genes, including Col I and Tnmd, a reasonable finding as collagen and Tnmd appear in significant amounts later in embryonic development [45-47]. Over time, from D0 to D3, TGF 32 treatment induced similar trends in $\mathrm{Scx}$, Col I, TGF 32 , and Tnmd expression by MSCs and TPCs (Figure 5B-E). Similar responsiveness of MSCs as TPCs to TGF $\beta 2$ treatments compared to control conditions (Figures $2 \mathrm{~A}$ and $3 \mathrm{~A}$ ) and over time (Figure 5B-E) supports our hypothesis and suggests MSCs have tenogenic potential.

In contrast to TGF $\beta 2$, FGF4 combinations downregulated Scx in MSCs (Figure 2A) and had no effect on TPCs (Figure 3A). FGF signaling seems necessary for embryonic tendon development $[33,38]$. Thus, it was surprising that FGF4 reduced tenogenic marker levels in MSCs. Perhaps differences in composition of transcriptional regulators in MSCs versus TPCs resulted in different signaling responses to the same cues. Potential effects of a heterogeneous progenitor cell population in MSCs should also be considered. Elucidation of these differences could lead to informed tenogenesis strategies using MSCs.

Differential baseline Eln expression levels and responses to TGF $\beta 2$ and TGF $\beta 2$ + loading by TPCs compared to MSCs are intriguing (Figures 4 and 5). Eln is important for adult tendon function, but little is known about its involvement in tendon development. In our earlier studies, Eln fibers were not detected in embryonic limb tendon [46], though tropoelastin was found in embryonic ligamentum flavum [48]. It would be interesting to investigate Eln influences and elaboration in embryonic tendon development.

\section{Conclusion}

In summary, we showed MSCs have tenogenic potential, based on similar gene expression and proliferation responses as TPCs when subjected to tenogenic treatments. Distinctly parallel trends in gene responses seen with MSCs compared to TPCs suggest the cells share certain molecular mechanisms of responses, which deserve further investigation. Future studies over longer time points could identify TGF $\beta 2$ combinations that effectively tenogenically differentiate stem cells, and may elucidate a chronological order of tendon marker expression during tenogenesis. Our findings suggest continued investigation of MSC function in relation to embryonic TPCs could contribute to advancements in tendon tissue regeneration strategies.
Note: This article is part of an 'Emerging Investigators' collection showcasing the work of early career investigators who have demonstrated growing leadership in the field of stem cells and regenerative medicine. Other articles in the series can be found online at http://stemcellres.com/series/emerginginvestigators

\section{Abbreviations}

BM: basal medium; Col I: collagen type I; D: day; E: embryonic day; Eln: elastin; FBS: fetal bovine serum; FGF: fibroblast growth factor; GFP: green fluorescent protein; GM: growth medium; MSC: mesenchymal stem cell; PBS: phosphate-buffered saline; qPCR: quantitative polymerase chain reaction; Scx: scleraxis; TGF: transforming growth factor; Tnmd: tenomodulin; TPC: tendon progenitor cell.

\section{Competing interests}

The authors declare that they have no competing interests.

\section{Authors' contributions}

This study was conceived, designed and coordinated by JPB and CKK. Cell harvest, experiments and assays were conducted by JPB and TVG. Statistical analyses of the results and interpretation of the data were completed by JPB, MS, NRS and CKK. The manuscript was written and revised by JPB, MS, NRS and CKK. All authors have read and approved the final version of the manuscript.

\section{Acknowledgements}

We thank Drs Clifford Tabin and Ronen Schweitzer for the Scx-GFP breeder mice, Dr Juan Taboas for his mechanical loader design, Dr Joseph Marturano and Zachary Schiller for technical assistance, and Dr Laura Hockaday for reading this manuscript. We gratefully acknowledge the following funding sources: NIH postdoctoral Award K12GM074869 (to NRS), NIH NIBIB Award P41EB002520 and NIH NIAMS Award R03AR061036 (to CKK).

Received: 4 June 2014 Revised: 7 November 2014

Accepted: 5 March 2015 Published online: 09 May 2015

\section{References}

1. Butler DL, Juncosa N, Dressler MR. Functional efficacy of tendon repair processes. Annu Rev Biomed Eng. 2004;6:303-29.

2. Chen J, Xu J, Wang A, Zheng M. Scaffolds for tendon and ligament repair: review of the efficacy of commercial products. Expert Rev Med Devices. 2009;6:61-73.

3. Yang G, Rothrauff BB, Tuan RS. Tendon and ligament regeneration and repair: clinical relevance and developmental paradigm. Birth Defects Res C Embryo Today. 2013;99:203-22.

4. Nirmalanandhan VS, Juncosa-Melvin N, Shearn JT, Boivin GP, Galloway MT, Gooch C, et al. Combined effects of scaffold stiffening and mechanical preconditioning cycles on construct biomechanics, gene expression, and tendon repair biomechanics. Tissue Eng Part A. 2009;15:2103-11.

5. Teh TK, Toh SL, Goh JC. Aligned fibrous scaffolds for enhanced mechanoresponse and tenogenesis of mesenchymal stem cells. Tissue Eng Part A. 2013:19:1360-72.

6. Subramony SD, Dargis BR, Castillo M, Azeloglu EU, Tracey MS, Su A, et al. The guidance of stem cell differentiation by substrate alignment and mechanical stimulation. Biomaterials. 2013;34:1942-53.

7. Kuo CK, Tuan RS. Mechanoactive tenogenic differentiation of human mesenchymal stem cells. Tissue Eng Part A. 2008;14:1615-27.

8. Thomopoulos S, Das R, Birman V, Smith L, Ku K, Elson EL, et al. Fibrocartilage tissue engineering: the role of the stress environment on cell morphology and matrix expression. Tissue Eng Part A. 2011;17:1039-53.

9. Yang PJ, Levenston ME, Temenoff JS. Modulation of mesenchymal stem cell shape in enzyme-sensitive hydrogels is decoupled from upregulation of fibroblast markers under cyclic tension. Tissue Eng Part A. 2012;18:2365-75.

10. Scott A, Danielson P, Abraham T, Fong G, Sampaio AV, Underhill TM. Mechanical force modulates scleraxis expression in bioartificial tendons. J Musculoskelet Neuronal Interact. 2011;11:124-32. 
11. Shearn JT, Kinneberg KR, Dyment NA, Galloway MT, Kenter K, Wylie C, et al. Tendon tissue engineering: progress, challenges, and translation to the clinic. J Musculoskelet Neuronal Interact. 2011;11:163-73.

12. Kuo CK, Marturano JE, Tuan RS. Novel strategies in tendon and ligament tissue engineering: advanced biomaterials and regeneration motifs. Sports Med Arthrosc Rehabil Ther Technol. 2010;2:20.

13. Hankemeier S, Keus M, Zeichen J, Jagodzinski M, Barkhausen T, Bosch U, et al. Modulation of proliferation and differentiation of human bone marrow stromal cells by fibroblast growth factor 2: potential implications for tissue engineering of tendons and ligaments. Tissue Eng. 2005;11:41-9.

14. Moreau JE, Chen J, Bramono DS, Volloch V, Chernoff H, Vunjak-Novakovic G, et al. Growth factor induced fibroblast differentiation from human bone marrow stromal cells in vitro. J Orthop Res. 2005;23:164-74.

15. Schweitzer R, Chyung JH, Murtaugh LC, Brent AE, Rosen V, Olson EN, et al. Analysis of the tendon cell fate using Scleraxis, a specific marker for tendons and ligaments. Development. 2001;128:3855-66.

16. Brown JP, Finley VG, Kuo CK. Embryonic mechanical and soluble cues regulate tendon progenitor cell gene expression as a function of developmental stage and anatomical origin. J Biomech. 2014;47:214-22.

17. Murchison ND, Price BA, Conner DA, Keene DR, Olson EN, Tabin CJ, et al Regulation of tendon differentiation by scleraxis distinguishes force-transmitting tendons from muscle-anchoring tendons. Development. 2007;134:2697-708.

18. Mello MA, Tuan RS. High density micromass cultures of embryonic limb bud mesenchymal cells: an in vitro model of endochondral skeletal development. In Vitro Cell Dev Biol Anim. 1999;35:262-9.

19. Haas AR, Tuan RS. Chondrogenic differentiation of murine C3H10T1/2 multipotential mesenchymal cells: II. Stimulation by bone morphogenetic protein-2 requires modulation of $\mathrm{N}$-cadherin expression and function. Differentiation. 1999;64:77-89.

20. Pittenger MF, Mackay AM, Beck SC, Jaiswal RK, Douglas R, Mosca JD, et al. Multilineage potential of adult human mesenchymal stem cells. Science. 1999:284:143-7.

21. DeLise AM, Fischer L, Tuan RS. Cellular interactions and signaling in cartilage development. Osteoarthritis Cartilage. 2000;8:309-34.

22. Johnstone B, Hering TM, Caplan Al, Goldberg VM, Yoo JU. In vitro chondrogenesis of bone marrow-derived mesenchymal progenitor cells. Exp Cell Res. 1998;238:265-72.

23. Tuli R, Tuli S, Nandi S, Huang X, Manner PA, Hozack WJ, et al. Transforming growth factor-beta-mediated chondrogenesis of human mesenchymal progenitor cells involves $\mathrm{N}$-cadherin and mitogen-activated protein kinase and Wnt signaling cross-talk. J Biol Chem. 2003;278:41227-36.

24. Shamis Y, Hewitt KJ, Carlson MW, Margvelashvilli M, Dong S, Kuo CK, et al. Fibroblasts derived from human embryonic stem cells direct development and repair of 3D human skin equivalents. Stem Cell Res Ther. 2011;2:10.

25. Schiller ZA, Schiele NR, Sims JK, Lee K, Kuo CK. Adipogenesis of adipose-derived stem cells may be regulated via the cytoskeleton at physiological oxygen levels in vitro. Stem Cell Res Ther. 2013;4:79.

26. Xu B, Song G, Ju Y, Li X, Song Y, Watanabe S. RhoA/ROCK, cytoskeletal dynamics, and focal adhesion kinase are required for mechanical stretch-induced tenogenic differentiation of human mesenchymal stem cells. J Cell Physiol. 2012;227:2722-9.

27. Schiele NR, Marturano JE, Kuo CK. Mechanical factors in embryonic tendon development: potential cues for stem cell tenogenesis. Curr Opin Biotechnol. 2013;24:834-40

28. Glass ZA, Schiele NR, Kuo CK. Informing tendon tissue engineering with embryonic development. J Biomech. 2014;47:1964-8.

29. Mikic B, Wong M, Chiquet M, Hunziker EB. Mechanical modulation of tenascin- $C$ and collagen-XII expression during avian synovial joint formation. J Orthop Res. 2000;18:406-15.

30. Mikic B, Johnson TL, Chhabra AB, Schalet BJ, Wong M, Hunziker EB. Differential effects of embryonic immobilization on the development of fibrocartilaginous skeletal elements. J Rehabil Res Dev. 2000;37:127-33.

31. Germiller JA, Lerner AL, Pacifico RJ, Loder RT, Hensinger RN. Muscle and tendon size relationships in a paralyzed chick embryo model of clubfoot. J Pediatr Orthoped. 1998;18:314-8.

32. Edom-Vovard F, Schuler B, Bonnin MA, Teillet MA, Duprez D. Fgf4 positively regulates scleraxis and tenascin expression in chick limb tendons. Dev Biol. 2002;247:351-66.
33. Brent AE, Braun T, Tabin CJ. Genetic analysis of interactions between the somitic muscle, cartilage and tendon cell lineages during mouse development. Development. 2005;132:515-28.

34. Molloy $T$, Wang $Y$, Murrell $G$. The roles of growth factors in tendon and ligament healing. Sports Med. 2003;33:381-94.

35. Carpenter JE, Thomopoulos S, Flanagan CL, DeBano CM, Soslowsky LJ. Rotator cuff defect healing: a biomechanical and histologic analysis in an animal model. J Shoulder Elbow Surg. 1998;7:599-605.

36. Kuo CK, Petersen BC, Tuan RS. Spatiotemporal protein distribution of TGF-betas, their receptors, and extracellular matrix molecules during embryonic tendon development. Dev Dyn. 2008;237:1477-89.

37. Pryce BA, Watson SS, Murchison ND, Staverosky JA, Dunker N, Schweitzer R Recruitment and maintenance of tendon progenitors by TGF signaling are essential for tendon formation. Development. 2009;136:1351-61.

38. Brent AE, Tabin CJ. FGF acts directly on the somitic tendon progenitors through the Ets transcription factors Pea3 and Erm to regulate scleraxis expression. Development. 2004;131:3885-96.

39. Pryce BA, Brent AE, Murchison ND, Tabin CJ, Schweitzer R. Generation of transgenic tendon reporters, ScxGFP and SCXAP, using regulatory elements of the scleraxis gene. Dev Dyn. 2007;236:1677-82.

40. Theiler K. The House Mouse: Atlas of Embryonic Development. The New York Medical Journal. New York: Springer-Verlag; 1989.

41. Hatakeyama Y, Tuan RS, Shum L. Distinct functions of BMP4 and GDF5 in the regulation of chondrogenesis. J Cell Biochem. 2004;91:1204-17.

42. Lejard V, Blais F, Guerquin MJ, Bonnet A, Bonnin MA, Havis E, et al. EGR1 and EGR2 involvement in vertebrate tendon differentiation. J Biol Chem. 2011;286:5855-67.

43. Kodama N, Sekiguchi S. The development of spontaneous body movement in prenatal and perinatal mice. Dev Psychobiol. 1984;17:139-50.

44. Pelton RW, Nomura S, Moses HL, Hogan BL. Expression of transforming growth factor beta 2 RNA during murine embryogenesis. Development. 1989;106:759-67.

45. Marturano JE, Xylas JF, Sridharan GV, Georgakoudi I, Kuo CK. Lysyl oxidase-mediated collagen crosslinks may be assessed as markers of functional properties of tendon tissue formation. Acta Biomater. 2014;10:1370-9.

46. Marturano JE, Arena JD, Schiller ZA, Georgakoudi I, Kuo CK. Characterization of mechanical and biochemical properties of developing embryonic tendon. Proc Natl Acad Sci U S A. 2013;110:6370-5.

47. Shukunami C, Takimoto A, Oro M, Hiraki Y. Scleraxis positively regulates the expression of tenomodulin, a differentiation marker of tenocytes. Dev Biol. 2006;298:234-47.

48. Brown JP, Lind RM, Burzesi AF, Kuo CK. Elastogenic protein expression of a highly elastic murine spinal ligament: the ligamentum flavum. PLoS One. 2012; $7:$ e38475

\section{Submit your next manuscript to BioMed Central and take full advantage of:}

- Convenient online submission

- Thorough peer review

- No space constraints or color figure charges

- Immediate publication on acceptance

- Inclusion in PubMed, CAS, Scopus and Google Scholar

- Research which is freely available for redistribution 